\title{
'The Prevalence of Occult Bladder Dysfunction Among Diabetic Subjects Attending An Diabetic Outpatient Clinic
}

\author{
B K Srinivasan ${ }^{1}$,Amar Needhi Ganesan ${ }^{2}$ \\ (Department Of Urology, Meenakshi Medical College/ MAHER University, Tamil Nadu ,India )
}

\begin{abstract}
Aim : To study the prevalence of occult bladder dysfunction among diabetic subjects between 18 to 60 years of age from Tamilnadu attending diabetic outpatient clinic.

Methodology : Prospective cohort study.

Results : Of 90 patients recruited, 65 (72.2\%) were males and 25 (27.8\%) were females. ). Overall 37 patients (41.1\%) had bladder dysfunction and 53 patients (58.9\%) did not have bladder dysfunction. In the urodynamic group, 35 of 45 (77.8\%) had bladder dysfunction as defined in the study. In the non-urodynamics group, only 2 of 45 patients (4.4\%) had bladder dysfunction. Age of the patients ranged from 20 to 58 years (mean age $\pm S D$ $45.6 \pm 8.12$ years). Male patients were slightly older than females $(46.23 \pm 8.0$ years in males against $44.2 \pm 8.1$ years in females). One half of the males and one fourth of the females had bladder dysfunction $(P=0.012)$ (table 3).

Conclusion : Bladder dysfunction is seen often (35/45) in patients with moderate LUTS than in asymptomatic patients (2/45) or those with mild LUTS. Bladder dysfunction is significantly associated with peripheral neuropathy and association with other microvascular complications like retinopathy and nephropathy is not significant.
\end{abstract}

Keyword: Diabetes, Bladder Dysfunction, Uroflometry, Ipss, Luts

\section{Introduction}

Diabetes is one of the most common chronic diseases affecting people world wide. The increasing prevalence of Diabetes all over the world is a major public health concern. The prevalence of diabetes in the adults will rise from 135 million in 1995 to 300 million by the year 2025, and more than $75 \%$ of the diabetics will reside in developing countries as compared to $62 \%$ in 1995. By 2025, India, China and the U.S will have the largest number of people with diabetics [1]. Several factors have contributed to the increasing burden of diabetes. These include a specific increase in risk factors for type 2 diabetes, such as increasing obesity [2, 3], lack of adequate physical activity [4], and life style and food changes induced by urbanization. Another factor contributing to apparent increase in the prevalence of diabetes has been the improvement in surveillance systems for diabetes, which has allowed better assessment of the true burden of diabetes [5]. Along with increased incidence of diabetes, complications caused by diabetes are also on the rise. Diabetes has its influence on multiorgan involvement in chronic diseases. Diabetes affects every organ due to macrovascular, microvascular and metabolic changes.

Diabetic cystopathy (DC) is a chronic known complication among diabetics with prevalence of $26 \%$ to 87\% [6]. It has a significant impact on day-to-day life, predisposes individuals to urinary tract infections (UTIs), potentiates renal complications and compromises optimum health. It is characterized by impaired bladder sensations, increased bladder capacity, decreased detrusor contractility and increased residual urine. Diabetic cystopathy develops insidiously and symptoms do not appear until the disease is well advanced. The classic symptoms of diabetic cystopathy which have been described have not always been observed in diabetic patient and these subjects often demonstrate variable symptom presentations. Initially the patient may be entirely asymptomatic, but demonstrate abnormalities on urodynamic study [7]. If bladder dysfunction is diagnosed earlier, corrective measures like strict glycemic control, changing the voiding pattern to timed voiding or double voiding may halt the deterioration of renal function, decrease the chance of urinary tract infection and halt progression of cystopathy further. Many authors have proposed that the diabetic cystopathy as a component of diabetic neuropathy which occurs in subjects with long standing diabetes. A weak association between glycemic control and neuropathic changes has been documented in both type 1 and type 2 diabetes. A possible link between deterioration of renal function and chronic asymptomatic bacteriuria in individuals with diabetes and bladder dysfunction also has been postulated [8, 9]. Lack of association between cystopathy and progression of diabetic nephropathy was shown in another study [10]. In this scenario, it is imperative for the treating physicians to diagnose bladder dysfunction in diabetics at an asymptomatic stage and institute early treatment for this condition to prevent or delay complications like recurrent urinary tract infection, urolithiasis, urinary incontinence and renal failure. 
Patients And Methods

\section{Headings}

\section{Research Question:}

1. What is the prevalence of occult bladder dysfunction among diabetic subjects between 18 to 60 years of age from Tamilnadu attending diabetic outpatient clinic?

2. What is the pattern of bladder dysfunction among the studied subjects?

3. Is there any association between the prevalence of bladder dysfunction between symptomatic and asymptomatic subjects?

4. Is there an association between bladder dysfunction and microvascular complications of diabetes?

Inclusion Criteria:

1. All Tamil speaking diabetic subjects between 18 to 60 years of age attending diabetic outpatient clinic.

Exclusion Criteria:

1. Previous surgery of urethra, prostate or bladder.

2. Previous pelvic procedures likely to cause bladder denervation like Hysterectomy and Abdominoperineal excision of rectum.

3. Neurological diseases likely to influence the lower urinary tract except autonomic neuropathy.

4. Overactive bladder, Bladder outlet obstruction or any other abnormal urodynamic findings.

5. Bladder calculus.

6. Bacterial and tuberculous cystitis

7. Medications like anticholinergics, diuretics, antidepressants and antipsychotics.

\section{Calculation of sample size:}

The sample size was calculated from a retrospective study analyzing the prevalence of bladder dysfunction among symptomatic diabetic patients who were referred and urodynamically evaluated in the department of urology for a period of three years. The prevalence of diabetic cystopathy was $40 \%$. The sample size required to find a prevalence of $40 \%$ with a precision of $\pm 10 \%$ and with a $95 \%$ confidence was 100 . Sample size was calculated using the formula: $4 \mathrm{pq} / \mathrm{d}^{2}$, where $\mathrm{p}$ is $40 \%, \mathrm{q}=1-\mathrm{p}=60 \%, \mathrm{~d}=10 \%$. The primary endpoint was to estimate the prevalence of occult bladder dysfunction.

\section{Methodology:}

The study was conducted in the diabetic outpatient clinic and urology department of Meenakshi medical college, Kanchipuram. This was prospective cohort study. Diabetic subjects of age group of 18 to 60 years attending the diabetic outpatient clinic were recruited for the study. Those who fulfill the inclusion and exclusion criteria were subjected for the study after written informed consent. They were asked to answer an international prostatic symptom score (IPSS) questionnaire (annexure 1). All subjects did a representative uroflowmetry. Post void residual urine was measured using abdominal ultrasound using prolate ellipsoid formula; Volume $(\mathrm{V})$ in $\mathrm{ml}=$ Length $\mathrm{x}$ Height $\mathrm{x}$ transverse diameter $\mathrm{x} \pi / 6$ or 0.53 . All maintained a bladder diary for 24 hours in their homes and returned the form during next visit. Those who have moderate lower urinary tract symptoms (LUTS) as indicated by (IPSS score $\geq 8$ ) or have a peak flow of less than $15 \mathrm{ml} / \mathrm{sec}$ at a voided volume more than $150 \mathrm{ml}$ were subjected for urodynamic evaluation. All the demographic and clinical data were recorded in the proforma (annexure 2). Evaluation of patients began with detailed history of duration of diabetes, duration of LUTS, nature of LUTS, presence of incontinence / hematuria / calculuria/ necroturia / urinary tract infection / voiding dysfunction/ instrumentation, symptoms of cardiac/ peripheral vascular / neurological diseases. Physical examination details as recorded by diabetologist (co-investigator) were also entered. Urological clinical assessment was done by the investigator. Evaluation for concomitant diabetic neuropathy, nephropathy and retinopathy was carried out in all patients. Estimation of serum creatinine, Fasting and 2-hours post prandial glucose, HbA1C, Fasting lipid profile, Microalbumin or 24-hour urinary proteins and urine microscopy was done for all. Urine culture and sensitivity was done for those who were subjected to urodynamics. Ultrasonogram of the abdomen and X-ray of the KUB region was done for those who had other abdominal symptoms, microhematuria and renal failure. Those who have asymptomatic bacteriuria were included in the study. Those who have positive culture with LUTS were excluded from the study. The pressureflow studies were done using medical measurement systems (MMS) UD 2000 equipment. Gentamicin Sulphate $160 \mathrm{mg}$ and Cefotaxime $1 \mathrm{~g}$ was given intravenously as prophylactic antibiotics for those who had normal renal function and those with renal failure respectively. No antibiotic prophylaxis was given after urodynamic study. Pretest residue was measured prior to urodynamic evaluation by placing two 6Fr infant feeding tubes. One of these tubes was used for filling as well as for intravesical pressure measurement. During cystometry in sitting posture, bladder was filled with physiological saline at $37^{\circ} \mathrm{C}$ at a filling rate of $50 \mathrm{ml} / \mathrm{min}$. First sensation of bladder filling $(\mathrm{ml})$, maximum cystometric capacity $(\mathrm{ml})$, detrusor overactivity (presence or absence), 
incontinence (presence or absence), and compliance $(\mathrm{cmH} 2 \mathrm{O})$ were assessed during filling phase. Maximum urinary flow (Qmax, $\mathrm{ml} / \mathrm{sec})$, Maximum intravesical pressure on voiding (cmH2O), Voided volume (ml), events like abdominal straining were noted during voiding phase. Abdominal pressure was recorded by using perforated rectal balloon catheter. Detrusor pressure was calculated by subtracting intraabdominal pressure from intravesical pressure. Detrusor pressure at maximum urinary flow rate (Pdet at Qmax, cm H2O) was measured to evaluate detrusor contractility. Methods, definitions and units were appropriate to the standards recommended by the international continence society. In those who did not undergo urodynamics, uroflowmetry, estimation of residual urine, bladder voiding efficiency (BVE) were the parameters evaluated to study bladder dysfunction. $\mathrm{BVE}$ was calculated by $\mathrm{BVE}=100 \% \mathrm{x}$ volume voided / (volume voided+ post void residue). BVE less than $75 \%$ was chosen as the cutoff point of bladder dysfunction.

Definition:

1) Urinary incontinence was defined if the complaint of any involuntary leakage of urine was present in which stress urinary incontinence (the complaint of involuntary leakage on effort on exertion, or on sneezing or coughing), or urge urinary incontinence (the complaint of involuntary leakage accompanied by or immediately preceded by urgency) were noted.

2) Normosensitive bladder - Volume at first sensation of $150-200 \mathrm{ml}$

3) Delayed first sensation - Appreciation of first sensation of filling at volume $\geq 250 \mathrm{ml}$ or greater than $50 \%$ of maximal cystometric capacity.

4) Detrusor overactivity - Involuntary phasic increase in detrusor pressure that was difficult to control or could not be controlled by patient resulting in incontinence or voiding.

5) Normal compliance - Filling detrusor pressure of $5-20 \mathrm{cmH} 2 \mathrm{O}$ in the absence of simultaneous detrusor contraction at maximum cystometric capacity.

6) Normal maximum cystometric capacity - Volume 350 to $600 \mathrm{ml}$, at which there was bladder contraction that resulted in voiding or patient discomfort.

7) Normal urinary flow rate - Catheterized urine flow rate of more than $12 \mathrm{ml} / \mathrm{sec}$.

8) Normal Pdet at Qmax - > $10 \mathrm{~cm} \mathrm{H} 2 \mathrm{O}$ or $<40 \mathrm{cmH} 2 \mathrm{O}$ during voiding with catheterized flow rate if more than $12 \mathrm{ml} / \mathrm{sec}$.

9) Normal post void residue $-50 \mathrm{ml}$

10) Hypocontractile detrusor - Pdet at Qmax less than $10 \mathrm{cmH} 2 \mathrm{O}$ or flat trace during voiding with or without abdominal straining.

11) Bladder outflow obstruction - Pdet at Qmax more than $40 \mathrm{cmH} 2 \mathrm{O}$ with catheterized urine flow rate less than $12 \mathrm{ml} / \mathrm{sec}$.

12) Bladder dysfunction (among those who had urodynamics): Presence of 2 or more of the following findings

a. First sensation of filling at volume more than $250 \mathrm{ml}$ or $50 \%$ of maximum cystometric capacity

b. Maximum cystometric capacity more than $600 \mathrm{ml}$

c. Compliance $>20 \mathrm{cmH} 2 \mathrm{O}$

d. Detrusor overactivity

e. Pdet at Qmax $<10$ / >40 $\mathrm{cmH} 2 \mathrm{O}$ with catheterized flow rate less than $12 \mathrm{ml} / \mathrm{sec}$

f. Post void residue more than $50 \mathrm{ml}$.

13) Bladder dysfunction (among those who did not have urodynamics): Presence of 2 or more of the following findings

a. Voided volume more than $600 \mathrm{ml}$ or less than $150 \mathrm{ml}$

b. Post void residue more than $50 \mathrm{ml}$

c. Bladder voiding efficiency less than $75 \%$

14) Diabetic nephropathy

a. Microalbuminuria: $\geq 30 \mathrm{mg} /$ day

b. Macroalbuminuria: $>300 \mathrm{mg} /$ day

\section{5) Diabetic retinopathy}

a. Presence of background, nonproliferative or proliferative retinopathy

b. History of laser retinal photocoagulation / vitreoretinal surgery in the course of diabetes

16) Diabetic neuropathy: Presence of more than 2 of the following elements

a. Biothesiometry $>20 \mathrm{mv}$

b. Monofilament $>4 \mathrm{~g}$

c. Deep tendon reflexes - absent or decreased 
d. Trophic ulcers

17) Overt Diabetic cystopathy: Presence of 3 or more of the following elements

a. First sensation of bladder filling at $>250 \mathrm{ml}$ or $50 \%$ of maximum cystometric capacity

b. Maximum cystometric capacity $>600 \mathrm{ml}$

c. Pdet at Qmax $<10 \mathrm{cmH} 2 \mathrm{O}$ at a catheterized urine flow rate of $12 \mathrm{ml} / \mathrm{sec}$

d. Post void residual urine $>50 \mathrm{ml}$

\section{Analysis}

All statistical analyses were performed using Statistical Package for the social Sciences (SPSS 11.0) for windows. Categorical data was presented using frequencies and percentage. Continuous data was described using mean \pm standard deviation or median and range. Associations between categorical variables were assessed using chi-square test with yates' correction or fisher's exact test. Continuous variables were compared using student ' $t$ ' tests and Mann-Whitney tests were used for non-normal data. A p-value less than 0.05 was considered statistically significant.

\section{Patients included in final analysis}

\section{Results}

A total of 90 patients were recruited for the study. Of 90 patients, $65(72.2 \%)$ were males and $25(27.8 \%)$ were females.

\section{Prevalence of bladder dysfunction}

Of 90 patients, 45 had urodynamic study according to the inclusion criteria and 45 others did not have urodynamic evaluation (non-urodynamics group). Over all 37 patients (41.1\%) had bladder dysfunction and 53 patients $(58.9 \%)$ did not have bladder dysfunction. In the urodynamic group, 35 of $45(77.8 \%)$ had bladder dysfunction as defined in the study. In the non-urodynamics group, only 2 of 45 patients (4.4\%) had bladder dysfunction. Age of the patients ranged from 20 to 58 years (mean age \pm SD $-45.6 \pm 8.12$ years). Male patients were slightly older than females ( $46.23 \pm 8.0$ years in males against $44.2 \pm 8.1$ years in females). One half of the males and one fourth of the females had bladder dysfunction $(\mathrm{P}=0.012)$ (table 3$)$.

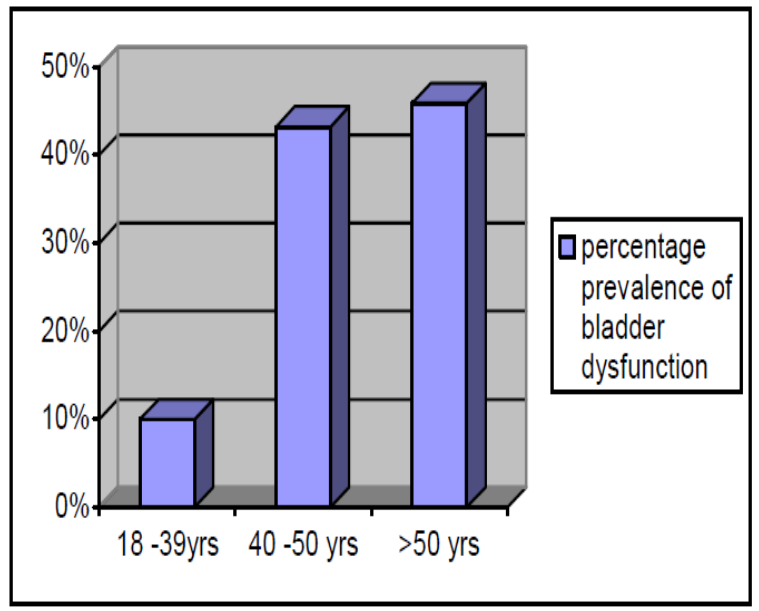

Figure 3: Prevalence

of bladder dysfunction

according to age

group.

Patients were categorized according to the age into three groups namely: 1) 18 to 39 yrs (21.1\%) 2) 4050 yrs $(48.9 \%)$ and 3) $>50 y r s ~(30 \%)$ (Figure 3). Bladder Figure 3: Prevalence of bladder dysfunction according to age group. dysfunction was seen more often in subjects with age $>50$ years $(45.9 \%)(\mathrm{P}=0.012)$. A few patients (7/90) were type 1 diabetics and majority (83/90) were type 2 diabetics. Bladder dysfunction was seen often in type 2 diabetics, however it was not statistically significant $(\mathrm{P}=0.610)$. Mean duration of diabetes was 6.7 years (range 1-25). Bladder dysfunction was seen in subjects with longer duration of diabetes (6 years Vs 3 years) $\mathrm{P}=0.042$. 
Table 3: Comparison of demographic features in bladder dysfunction

\begin{tabular}{llll}
\hline Variable & \multicolumn{2}{l}{ Bladder dysfunction } & \multirow{2}{*}{ P value } \\
\cline { 2 - 3 } & Yes & No & \\
\hline Age (years) & & & \\
Males $^{\S}$ & $49.0 \pm 8.9$ & $43.3 \pm 8.6$ & $0.001^{* *}$ \\
Females $^{\S}$ & $32(49.2 \%)$ & $33(50.8 \%)$ & $0.012^{*}$ \\
Type I Diabetes $^{\S}$ & $5(20 \%)$ & $20(80 \%)$ & \\
Type II Diabetes $^{\S}$ & $3(42.9 \%)$ & $4(57.1 \%)$ & 0.610 \\
Duration of diabetes (years) $^{\ddagger}$ & $3(1-25)$ & $3(1-25)$ & $0.042^{*}$ \\
\hline
\end{tabular}

$\dagger-$ mean \pm standard deviation

$\$$ - median (range)

$\S$ - percentage

* - significant

** - Highly significant

Bladder dysfunction was seen 10 (18.9\%), 24(70.6\%) and 3(100\%) in those who have mild, moderate and severe LUTS by IPSS $(\mathrm{P}<0.001)$ as denoted in table 4 . There was no correlation between IPSS and the severity of bladder dysfunction. Bladder dysfunction was seen more often in those who complained of LUTS during questioning $(\mathrm{P}=0.05)$.

Table 4: Comparison of Frequency -Volume chart (bladder diary), uroflowmetry \& Bladder voiding efficiency in bladder dysfunction

\begin{tabular}{|c|c|c|c|c|}
\hline \multirow{2}{*}{\multicolumn{2}{|c|}{ Variable }} & \multicolumn{2}{|c|}{ Bladder dysfunction } & \multirow{3}{*}{$\begin{array}{l}\text { P value } \\
<0.001^{* *}\end{array}$} \\
\hline & & Yes & No & \\
\hline \multirow[t]{3}{*}{ IPSS $^{\S}$} & Mild & $10(18.9 \%)$ & $43(81.1 \%)$ & \\
\hline & Moderate & $24(70.6 \%)$ & $10(29.4 \%)$ & \\
\hline & Severe & $3(100 \%)$ & 0 & \\
\hline \multirow[t]{2}{*}{ LUTS $^{\S}$} & Absence & $12(30 \%)$ & $28(70 \%)$ & $0.05^{*}$ \\
\hline & Presence & $25(50 \%)$ & $25(50 \%)$ & \\
\hline \multicolumn{2}{|l|}{ Duration of LUTS (months) $)^{\ddagger}$} & $6(0-24)$ & $0(0-36)$ & $0.002 * *$ \\
\hline \multicolumn{2}{|l|}{ Day time frequency (times) } & $7(5-12)$ & $6(4-16)$ & 0.264 \\
\hline \multicolumn{2}{|l|}{ Night time frequency (times) } & $2(0-6)$ & $1(0-5)$ & $0.007 *$ \\
\hline \multicolumn{2}{|l|}{ 24-hrs Fluid intake $(\mathrm{ml})$} & $\dagger 2750 \pm 1151$ & $2712 \pm 964$ & 0.870 \\
\hline \multicolumn{2}{|l|}{ 24-hrs urine volume (ml) ${ }^{\dagger}$} & $2858 \pm 1337$ & $2386 \pm 708$ & $0.05^{*}$ \\
\hline \multicolumn{2}{|l|}{$\begin{array}{l}\text { Maximum bladder volume(ml) } \\
\text { (bladder diary) }{ }^{\dagger}\end{array}$} & $582.30 \pm 218$ & $502 \pm 196$ & 0.079 \\
\hline \multirow{2}{*}{$(0 \cos$} & $<15 \mathrm{ml} / \mathrm{sec}$ & $35(77.7 \%)$ & $10(22.2 \%)$ & $<0.001^{* *}$ \\
\hline & $<15 \mathrm{ml} / \mathrm{sec}$ & $2(4.4 \%)$ & $43(95.6 \%)$ & \\
\hline Voided volume & $(>600 \mathrm{ml})$ & $19(52.8 \%)$ & $17(47.2 \%)$ & $0.05^{*}$ \\
\hline During uroflow ${ }^{\S}$ & $(>600 \mathrm{ml})$ & $18(33.3 \%)$ & $36(66.7 \%)$ & \\
\hline \multirow[t]{2}{*}{ Post void residue ${ }^{\S}$} & $>50 \mathrm{ml}$ & $14(70 \%)$ & $6(30 \%)$ & $0.003 *$ \\
\hline & $<50 \mathrm{ml}$ & $23(32.9 \%)$ & $47(67.1 \%)$ & \\
\hline Post void residue $(\mathrm{ml})^{\dagger}$ & & $175.9 \pm 232.4$ & $52.4 \pm 58.6$ & $0.007^{*}$ \\
\hline Bladder voiding efficiency (\%) & & $87.7 \pm 17.3$ & $94.56 \pm 5.13$ & $0.025^{*}$ \\
\hline
\end{tabular}

$\dagger-$ mean \pm standard deviation

$\$$ - median (range)

$\S$ - percentage

* - significant $* *$ - Highly significant

Bladder dysfunction was seen in those who had LUTS for 6 months or more $(\mathrm{P}=0.002)$. Bladder dysfunction was seen more often in those who had nocturnal frequency $(\mathrm{P}=0.007)$. Diabetics with bladder 
dysfunction has slightly increased intake of fluid, but the difference between the groups was not significant $(\mathrm{P}=0.870)$. However there was significant difference between the group with bladder dysfunction and the group that did not have dysfunction in 24-hr urine output. It could be attributed to hyperosmolar state in poorly controlled diabetes, as evident by the increase in mean $\mathrm{HbA1C}$ level $(8.46 \pm 2.03 \%)$ in those with bladder dysfunction. However it was not statistically significant from HbA1C levels seen in group which did not have bladder dysfunction. The mean 24-hr urine output in subjects with bladder dysfunction was $2858 \pm 13 \mathrm{ml}$ and it was $2386 \pm 70 \mathrm{ml}$ in those who did not have dysfunction $(\mathrm{P}=0.05)$.

There was a significant difference between subjects with bladder dysfunction and Those who did not have bladder dysfunction, when their peak urine flow was less than $15 \mathrm{ml} / \mathrm{sec}(\mathrm{P}=<0.001)$, voided volume was more than $600 \mathrm{ml}(\mathrm{P}=0.05)$ and post void residue was more than $50 \mathrm{ml}(\mathrm{P}=0.003)$. When the post void residual urine values was individually computed for both groups, patients with bladder dysfunction had significantly different high mean volumes as compared to those who did not have bladder dysfunction $(175.9 \pm 232.4 \mathrm{ml} \mathrm{Vs}$ $52.4 \pm 58.6 \mathrm{ml})(\mathrm{P}=0.007)$. Mean bladder voiding efficiency was also significantly lesser in dysfunction group than in the group that did not have bladder dysfunction $(87.7 \pm 17 \%$ Vs $94.56 \pm 5.13 \%)(\mathrm{P}=0.025)$.

\section{Prevalence of various urodynamic abnormalities in dysfunction group}

The prevalence of various urodynamic abnormalities in detrusor activity, first sensation, maximum cystometric capacity, bladder compliance, bladder outflow obstruction (Pdet at Qmax of $\geq 40 \mathrm{cmH} 2 \mathrm{O}+$ catheterized urine flow rate of $12 \mathrm{ml} / \mathrm{sec}$ ), hypocontractile detrusor (Pdet at Qmax $<10 \mathrm{cmH} 2 \mathrm{O}$ ) and increased post void residue ( $>50 \mathrm{ml}$ ) among those who had bladder dysfunction in the urodynamic group is shown in table 5. Increased maximum cystometric capacity was the commonest abnormality seen.

Table 6. Prevalence of various urodynamic abnormalities

\begin{tabular}{|c|c|c|c|}
\hline \multicolumn{3}{|c|}{ Urodynamic abnormalities } & Prevalence - No (\%) \\
\hline \multicolumn{3}{|c|}{ Detrusor overactivity } & $14(31.1)$ \\
\hline $\begin{array}{l}\text { Increased } \\
\text { capacity }\end{array}$ & maximum & cystometric & $30(66.6$ \\
\hline \multicolumn{3}{|c|}{ Decreased bladder compliance } & $15(33.3)$ \\
\hline \multicolumn{3}{|c|}{ Delayed first sensation } & $15(33.3)$ \\
\hline \multicolumn{3}{|c|}{ Bladder outflow obstruction } & $15(33.3)$ \\
\hline \multicolumn{3}{|c|}{ Increased post void residue } & $25(55.6)$ \\
\hline
\end{tabular}

\section{Comparison of urodynamic parameters in bladder dysfunction}

Delayed onset of first sensation of filling (>250ml or $50 \%$ of maximum cystometric capacity), increased maximum cystometric capacity $(>600 \mathrm{ml})$, peak catheterized flow rate (Qmax Uro), post void residual urine ( $>50 \mathrm{ml}$ ) and abnormal detrusor contraction (Pdet Qmax $<10$ or $>40 \mathrm{cmH} 2 \mathrm{O}$ ) were significantly different between those who had bladder dysfunction and those who did not have dysfunction. However detrusor overactivity, compliance, detrusor pressure at peak catheterized flow rate (Pdet at Qmax Uro) was not significantly different between the two groups as denoted in table 7 .

Table 7- Significance of urodynamic parameters in bladder dysfunction

\begin{tabular}{|c|c|c|c|c|}
\hline \multicolumn{2}{|l|}{ Variable } & \multicolumn{2}{|c|}{ Bladder dysfunction } & \multirow[t]{2}{*}{ P value } \\
\hline & & Yes & No & \\
\hline \multirow[t]{2}{*}{ First sensation $^{\S}$} & $>250 \mathrm{ml}$ & $15(100 \%)$ & $\mathbf{0}$ & $0.009 *$ \\
\hline & $>250 \mathrm{ml}$ & $20(66.7 \%)$ & $10(33.3 \%)$ & \\
\hline First sensation & $>50 \%$ & 31 & 53 & $0.004 *$ \\
\hline (of cystometric & $>50 \%$ & 6 & $\mathbf{0}$ & \\
\hline \multicolumn{5}{|l|}{${\text { capacity })^{\S}}^{\S}$} \\
\hline \multicolumn{2}{|c|}{ Detrusor overactivity } & $13(92.9 \%)$ & $1(7.1 \%)$ & 0.102 \\
\hline Maximum & $(>600 \mathrm{ml})$ & $19(52.8 \%)$ & $17(47.8 \%)$ & $0.05^{*}$ \\
\hline cystometric & $(>600 \mathrm{ml})$ & $18(33.3 \%)$ & $36(66.7 \%)$ & \\
\hline \multicolumn{5}{|l|}{ capacity $^{\S}$} \\
\hline \multirow[t]{2}{*}{ Compliance $^{\S}$} & $>20 \mathrm{cmH} 2 \mathrm{O}$ & $14(93.3 \%)$ & $1(6.7 \%)$ & 0.077 \\
\hline & $>20 \mathrm{cmH} 2 \mathrm{O}$ & $21(70 \%)$ & $9(30 \%)$ & \\
\hline \multicolumn{2}{|c|}{$\operatorname{Qmax}^{\text {Uro }}(\mathrm{ml} / \mathrm{sec})^{\dagger}$} & $10.7 \pm 4.3 *$ & $14.4 \pm 3.6$ & $0.016 *$ \\
\hline \multicolumn{2}{|c|}{ Pdet at Qmax ${ }^{\text {Uro }}(\mathrm{cm} \mathrm{H2O})^{\dagger}$} & $41.4 \pm 21.5$ & $32.4 \pm 15.8$ & 0.401 \\
\hline
\end{tabular}


'The Prevalence Of Occult Bladder Dysfunction Among Diabetic Subjects ...

\begin{tabular}{|c|c|c|c|}
\hline Pdet Qmax $<10$ or $>40^{\S}$ & $23(88.5 \%)$ & $3(11.5 \%)$ & $0.05^{*}$ \\
\hline \multirow[t]{2}{*}{${\text { Post void residue }(\mathrm{ml})^{\dagger}}^{\dagger}$} & $175.9 \pm$ & \multirow[t]{2}{*}{$52.4 \pm 58.6$} & \multirow[t]{2}{*}{$0.007 *$} \\
\hline & 232.49 & & \\
\hline
\end{tabular}

$\dagger-$ mean \pm standard deviation

$\$-$ median (range)

$\S$ - percentage

* - significant $* *$ - Highly significant

\section{Comparison of clinical parameters in bladder dysfunction}

Among the clinical parameters studied, presence of peripheral neuropathy was the only parameter found to be significantly different between the bladder dysfunction group and the group that did not have bladder dysfunction $(\mathrm{P}=0.018)$ (table 8). Presence of retinopathy, nephropathy, blood pressure values, body mass index (BMI) and associated diseases were not significantly different between the two groups. When biothesiometry and monofilament assessment was correlated individually with presence or absence of bladder dysfunction, only abnormal monofilament values were significantly different between the bladder dysfunction and the group without bladder dysfunction $(\mathrm{P}=0.022)$.

Table 8: Comparison of clinical parameters in bladder dysfunction

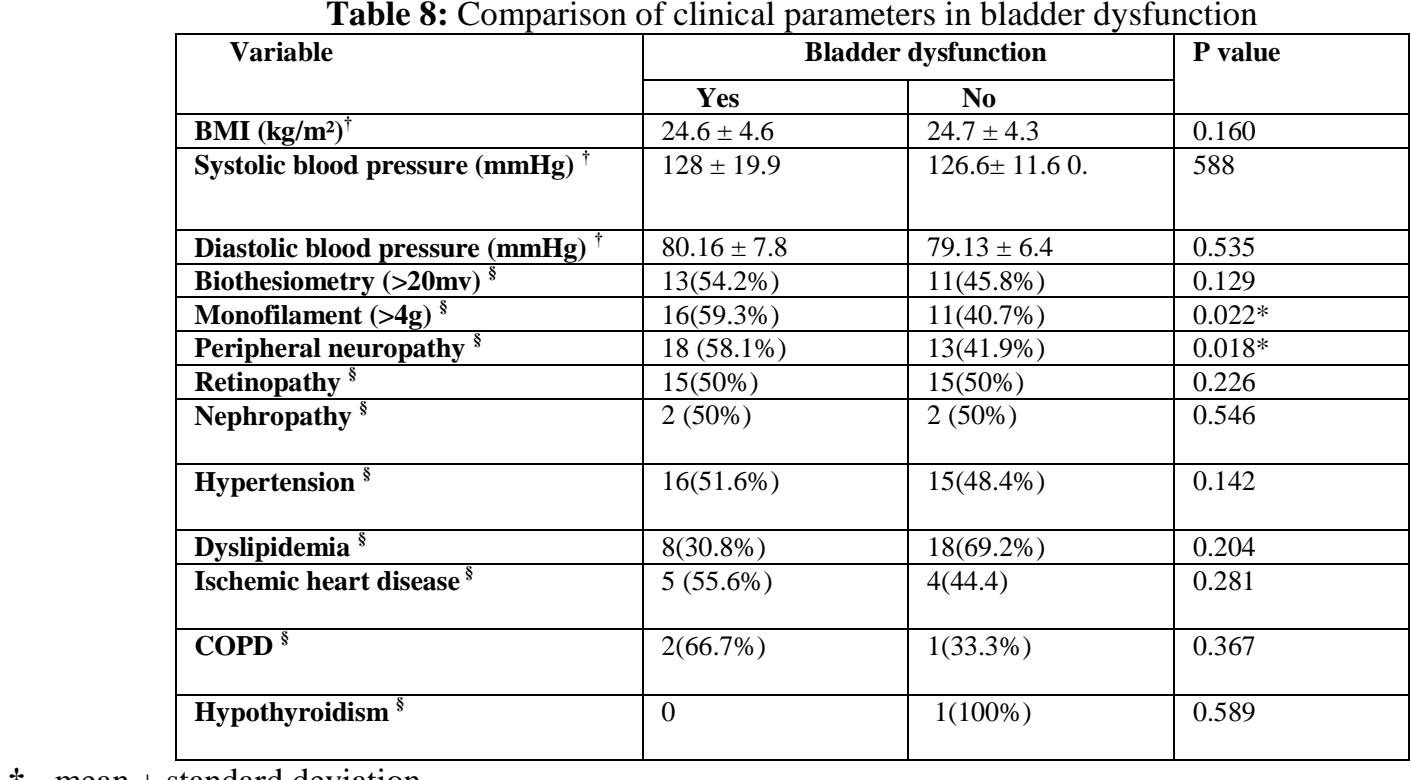

$\dagger$ - mean \pm standard deviation,

$\S$ - percentage, $*$ - significant

\section{Comparison of biochemical parameters with bladder dysfunction}

Similarly there was no difference between the two groups in blood sugar levels, HbA1C, Microalbumin and lipid profile (table 9). Serum creatinine was the only biochemical parameter found to be significantly different between the bladder dysfunction group and the group which did not have bladder dysfunction. Only two of the patients with bladder dysfunction had clinical renal failure.

Table 9: Comparison of biochemical parameters with bladder dysfunction

\begin{tabular}{|c|c|c|c|}
\hline \multirow[t]{2}{*}{ Variable } & \multicolumn{2}{|c|}{ Bladder dysfunction } & \multirow[t]{2}{*}{ P value } \\
\hline & Yes & No & \\
\hline $\mathrm{AC}(\mathrm{mg} \%)^{\dagger}$ & $140 \pm 49$ & $145 \pm 45$ & 0.678 \\
\hline $\mathbf{P C}(\mathbf{m g ~ \%})^{\dagger}$ & $224 \pm 78$ & $214 \pm 76$ & 0.620 \\
\hline Serum creatinine $(\mathrm{mg} \%)^{\dagger}$ & $0.97 \pm 0.24$ & $0.86 \pm 0.14$ & 0.018 \\
\hline $\operatorname{HbA1C}(\%)^{\dagger}$ & $8.46 \pm 2.03$ & $8.43 \pm 1.85$ & 0.420 \\
\hline Microalbumin (mg) ${ }^{\dagger}$ & $69.05 \pm 88.46$ & $46.6 \pm 79.15$ & 0.312 \\
\hline${\text { Cholesterol }(\mathrm{mg} \%)^{\dagger}}^{\dagger}$ & $178.14 \pm 42.36$ & $182.15 \pm 44.37$ & 0.914 \\
\hline
\end{tabular}


'The Prevalence Of Occult Bladder Dysfunction Among Diabetic Subjects ...

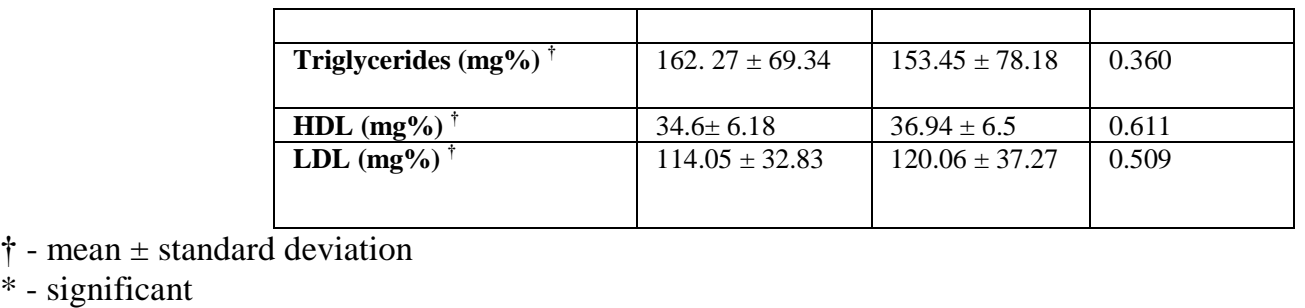

\section{Discussion}

In the previous studies, according to the diagnostic methods, criteria and patient characteristics, the frequency of diabetic cystopathy varies from $26 \%$ to $87 \%[6,7,9,79]$. The varied frequency of diabetic bladder dysfunction reported in the literature is due to varied definitions adopted and different combinations of investigations used by the authors. Frimodt-Moller et al first reported detailed clinical characteristics of bladder dysfunction in unselected diabetic patients. They characterized diabetic cystopathy as loss of sensation detected by elevated

electrical bladder perception threshold which was found in $38 \%$ of patients [80].According to the criteria proposed by Kahan et al [77], which defined diabetic cystopathy as an increase in bladder capacity to more than $400 \mathrm{ml}$ with flat trace on cystometry, $36 \%$ of diabetic subjects had diabetic cystopathy. Ueda et al [39] reported that $32 \%$ of diabetic patients had diabetic cystopathy using the criterion that bladder capacity exceeding $500 \mathrm{ml}$ was abnormal. In our study, with criteria for overt diabetic cystopathy which included maximum cystometric capacity $(>600 \mathrm{ml})$, Pdet at Qmax $<10 \mathrm{cmH} 2 \mathrm{O}$ at catheterized flow rate of less than $12 \mathrm{ml} / \mathrm{sec}$ and impaired first sensation $(>250 \mathrm{ml}$ or $50 \%$ of cystometric capacity) and PVR $\geq 50 \mathrm{ml}$, overt diabetic cystopathy was found in $7 / 45(15.5 \%)$ patients. When all the criteria for bladder dysfunction were applied, Overall prevalence of it

was noted in $41.1 \%$ of the patients. Kebapci et all has shown that IPSS was not different between those who have bladder dysfunction and those who did not have bladder dysfunction in their study of 54 type 2 diabetic patients. It was also not different among those who had diabetic cystopathy and those who had other bladder dysfunction [82]. In our study mean IPSS was significantly different among the bladder dysfunction (9.7 $\pm 6)$ and non dysfunction group $(4.3 \pm 3.3)(\mathrm{P}<0.001)$. However we could not establish a correlation between the IPSS and severity of bladder dysfunction. There was highly significant difference in peakflow among the bladder dysfunction group $(17.13 \mathrm{ml} / \mathrm{sec})$ and group without bladder dysfunction $(25.02 \pm 8.32 \mathrm{ml} / \mathrm{sec})$ $(\mathrm{P}<0.001)$. The same difference noted for post void residue $>50 \mathrm{ml}$ between these groups $(70 \% \mathrm{Vs} 50 \%)(\mathrm{P}=$ 0.003). Thus its should be possible to predict the chances of bladder dysfunction in diabetics with basic tools of evaluation of lower urinary tract like uroflowmetry, estimation of post void residue and IPSS (peak flow less than $17 \mathrm{ml} / \mathrm{sec}$, IPSS $>10$ and PVR $>50 \mathrm{ml}$ ). However the sample size should be larger, to get more significant and consistent prediction of bladder dysfunction. A number of clinical studies have reported detrusor overactivity as the most frequent finding, ranging from $39 \%$ to $61 \%$ of diabetic patients [88]. Detrusor overactivity can be due to bladder outflow obstruction or neurologic disease and moreover, it is common among elderly incontinent subjects [39]. Incontinence due to detrusor overactivity among women in the general population increases with age, starting at a prevalence / incidence of $20-30 \%$ in young-adult life ( $<40$ years), rising to $30-40 \%$ in middle age (40-60 years), and 30-50\% in the elderly (>60 years) [88, 89]. Similarly detrusor overactivity is also of high prevalence among elderly men with prostatic enlargement [90]. Men with diabetes have an increased risk of developing LUTS, reported to be $25-100 \%$ higher than in the general male population [91]. In our study, in the absence of neurological disease other than diabetic neuropathy, among patients of bladder dysfunction, detrusor overactivity was seen in 13 patients (females -4, males -9) with prevalence of $29 \%$. Of them, isolated detrusor overactivity, detrusor overactivity associated with BOO and associated with cystopathy was seen in $38.5 \%, 38.5 \%$ and $23 \%$ of patients respectively. Among females with detrusor overactivity, it was seen as an isolated abnormality in two and was associated with cystopathy in others.

Among males with detrusor overactivity, it was seen isolated in 3, associated with BOO in 5 and associated with cystopathy in one. Mean age of males with detrusor overactivity was 51.4 years. Mean age of females with detrusor overactivity was 47.7 years. Within the male population the most important differential diagnosis, and the one that frequently coexists with diabetic cystopathy is BOO [92]. Kaplan et al found that bladder outflow obstruction was seen in 66 men (prevalence of 36\%) among their 182 patients who were evaluated with urodynamics [35]. Bladder outflow obstruction was seen 15 (33.3\%) patients in our study. All except one were men. A 55 years old postmenopausal lady had BOO. The mean age of all men was them 47.2 years (range $39-58$ years). 3 patients with BOO also had features of diabetic cystopathy. 5 had associated detrusor overactivity.

The patients who had bladder dysfunction had significant difference in maximum bladder volume as recorded in the bladder diary and higher 24-hr urinary volume (urine output). This could also be useful to 
predict bladder dysfunction provided the hyperosmolar state due to poorly controlled diabetes is ruled out. In our study both the bladder dysfunction and non-dysfunction groups had raised HbAlC levels indicating inadequate glycemic control. This could be the reason for larger urinary volumes seen among subjects with bladder dysfunction. Kepabci et al have shown in their study that higher HbA1C levels were associated with higher prevalence of bladder dysfunction even though it was not statistically significant. There is well established correlation between bladder dysfunction and diabetic neuropathy [93]. The presence of abnormalities on neurological examination has been shown to predict bladder dysfunction. More than $85 \%$ of patients with positive sacral cord signs had abnormalities of detrusor contractions in one study [35]. In another study on 29 diabetic patients, 38 of whom had voiding dysfunction as defined by the presence of an abnormality in either flow rate, flow pattern, or post void residue, voiding dysfunction was strongly correlated with presence of neuropathy $(\mathrm{P}<0.001)$ [41]. Overall, a correlation ranging from $75 \%$ to $100 \%$ have been reported in previous studies [76]. In our study, 18 of the 31 (58.1\%) patients with clinical evidence of peripheral neuropathy in lower limbs had bladder dysfunction, whereas only 19 of the 59 patients without neuropathy had bladder dysfunction $(\mathrm{P}=0.018)$. Presence of overt peripheral neuropathy could be used to predict the bladder dysfunction. Esteghamati et al showed an inverse correlation between retinopathy and detrusor instability in an urodynamic study done in asymptomatic diabetic subjects [86].

About half the patients with diabetic cystopathy showed signs of retinopathy in study of 124 diabetic subjects [80]. In our study, prevalence of retinopathy was 33\%.There was no significant difference between bladder dysfunction group and group which did not have bladder dysfunction $(\mathrm{P}=0.226)$. Bladder dysfunction was seen 22 patients without retinopathy. In a small study on 17 insulin dependent diabetic patients with median age of 45 years (27-67years) and mean diabetes duration of 23 years (14-44years) who suffered from diabetic nephropathy, no association was found between diabetic cystopathy and progression of nephropathy [10]. The frequency of nephropathy was 19\% among patients with diabetic cystopathy in the study by Frimodt-Moller [80]. Serum creatinine was highly significant among those with nephropathy and cystopathy $(\mathrm{P}<0.001)$. In our study serum creatinine was significantly different in bladder dysfunction group and those who did not have bladder dysfunction. However there was no significant correlation between bladder dysfunction and nephropathy. There were two patients with renal failure in the bladder dysfunction group, whose altered renal function is probably because of diabetic nephropathy rather than cystopathy as both had macroalbuminuria. As denoted by Kaplan et al [35], classical diabetic cystopathy was not the common abnormality seen among diabetic subjects; we found a variety of urodynamic abnormalities as denoted in the table 6 . The present study was one to evaluate the prevalence of bladder dysfunction of probably asymptomatic diabetic subjects who had never come to urology department owing to their lower urinary tract symptoms and were recruited from a diabetic clinic of an endocrinology department. Although most of the patients in our study were urologically asymptomatic or had mild symptoms as evidenced by the low mean IPSS, majority (35/45) of those who had urodynamic evaluation had atleast one urodynamic abnormality. 7 (15.5\%) had overt diabetic cystopathy and they never bothered to the extent of seeking urological advise. It indicates the very innocuous and occult nature of development of bladder dysfunction in diabetics. Unless they were questioned appropriately and diagnosed, they would have developed the classical cystopathy and its complications. It's also worrisome to anticipate such complications happening in the group who did not have urodynamic evaluation. However considering ethical reasons, invasive urodynamic evaluation was not done in them. If we are able to predict the bladder dysfunction consistently using the uroflowmetry, post void residue and IPSS, it can be applied for screening the asymptomatic subjects. If any abnormality is detected during initial screening, they can be subjected to further urodynamic study, which is a more sensitive and accurate method of evaluating voiding function. The findings in this study suggest the development of diabetic bladder dysfunction begins far before symptoms appear or when symptoms are mild in nature. It highlights the importance of early screening for bladder dysfunction, especially for those who are at risk.

From this study, it can be concluded that

\section{Conclusions}

1) The prevalence of bladder dysfunction in diabetic subjects between 18 to 60 years of age from Tamilnadu attending a diabetic clinic was $41.1 \%$.

2) Abnormal urodynamic findings other than diabetic cystopathy are commonly seen among the diabetic subjects.

3) IPSS, Uroflowmetry and estimation of post void residual urine might be useful to screen the diabetic subjects before urodynamic evaluation to diagnose and characterize bladder dysfunction.

4) Bladder dysfunction is seen often (35/45) in patients with moderate LUTS than in asymptomatic patients (2/45) or those with mild LUTS.

5) Bladder dysfunction is significantly associated with peripheral neuropathy and association with other microvascular complications like retinopathy and nephropathy is not significant. 


\section{Limitations}

1) Small sample size of one particular community (Tamil speaking local people) may not represent the whole of the population of diabetic subjects in the country. Regional difference among various subjects and its implication on bladder dysfunction is not addressed. Larger sample size involving participation of subjects from varied geographical distribution might be useful to overcome the regional bias.

2) To elucidate the prediction of the chance of developing bladder dysfunction by the parameters studied may give erroneous value, particularly in the small present population of diabetic subjects.

3) We have only evaluated for peripheral somatic neuropathy (in the extremities) and the presence of autonomic neuropathy was not evaluated.

4) Some of the abnormal findings may normally occur with aging. However, because of ethical issues in performing invasive urodynamic studies on asymptomatic or mildly symptomatic patients, there was no real representation of bladder findings from the asymptomatic group even though calculation of bladder voiding efficiency was used to predict voiding dysfunction in them. Urodynamic evaluation in these subjects can throw light on the true prevalence of asymptomatic bladder dysfunction and can be detected at a very early stage.

5) The lack of associations between some of the parameters evaluated in this study might be due to the relatively small sample size and the consequent low power of the study. Studies with larger sample sizes can be performed in future to more accurately predict the bladder dysfunction. It should be possible to exactly predict the chances of bladder dysfunction using mathematical models with application of simple and clinically applicable measurements like uroflowmetry, post void residue and IPSS.

\section{Bibliography}

[1]. King H, Aubert RE, Herman WH: Global burden of diabetes, 1995-2025: prevalence, numerical estimates, and projections. Diabetes Care 1998 Sep; 21(9):1414-31.

[2]. Ford ES, Williamson DF, Liu S. Weight change and diabetes incidence: findings from national cohort studies of U.S adults. Am J Epidemiology 1997; 146:214-222.

[3]. Bjorntorp P. Obesity. Lancet 1997; 350: 423-426.

[4]. National Center for Chronic Disease Prevention and Health Promotion. Physical activity and health: a report of the surgeon general. Atlanta: Centers for Disease Control and Prevention,1996.

[5]. National Center for Chronic Disease Prevention and Health Promotion. Diabetes surveillance, 1993.Atlanta: Centers for Disease Control and Prevention, 1993

[6]. Frimodt- moller C: Diabetic cystopathy. A review of Urodynamic and clinical features of neurogenic bladder dysfunction in diabetes mellitus. Dan Med Bull, 25; 49, 1978

[7]. Ellenberg M, Weber H. The incipient asymptomatic diabetic bladder. Diabetes 1967; 16: 331-335.

[8]. Geerlings S, Stolk RP, Camps MJL, Netten PM, Collet JT, Schneeberger PM, et al. Consequences of asymptomatic bacteriuria in women with diabetes mellitus. Arch Intern Med 2001; 161:1421-7.

[9]. Faerman I, Maler M, Jadinsky M et al. Asymptomatic neurogenic bladder in juvenile diabetics. Diabetologia 1971; 7: 168-72.

[10]. Torffvit.O, Agardh.CD, Mattiasson A. Lack of association between cystopathy and progression of diabetic nephropathy in insulindependent diabetes mellitus. Scand J Urol Nephrol 1997. 31; 365-369.

[11]. Thor KB, Morgan KB, Morgan C, Nadelhaft I, Houston M, deGroat WC.Organization of afferent and efferent pathways in the pudendal nerve of the female cat. J Comp Neurol.1989 Oct 8; 288(2):263-79.

[12]. Morgan C, Nadelhaft I, deGroat WC. The distribution within the spinal cord of visceral primary afferent axons carried by the lumbar colonic nerve of the cat. Brain Res. 1986 Nov 19; 398(1):11-7.

[13]. Janig W, Morrison JF. Functional properties of spinal visceral afferents supplying abdominal and pelvic organs, with special emphasis on visceral nociception. Prog Brain Res. 1986; 67:87-114.

[14]. deGroat WC; Spinal cord projections and neuropeptides in visceral afferent neurons. Prog Brain Res. 1986; 67:165-87.

[15]. McMahon SB, Abel C: A model for the study of visceral pain states: Chronic inflammation of the chronic decerebrate rat urinary bladder by irritant chemicals. Pain 1987; 28:109.

[16]. Araki I, de Groat WC: Synaptic modulation associated with developmental reorganization of visceral reflex pathways. J Neurosci $1997 ; 17: 8402$ 